\title{
Uma leitura comunicacional da estratégia nas organizações
}

\author{
Victor Márcio Laus Reis Gomes
}

Resumo: Este artigo apresenta uma abordagem comunicacional para a compreensão da estratégia organizacional. Consagrada como objeto de abordagens administrativas e econômicas, a estratégia foi investigada a partir de uma perspectiva epistêmico-metodológica que privilegia a comunicação, e foi fundamentada na teoria sistêmica de Niklas Luhmann, na dimensão social da linguagem e na teoria discursiva de Eliseo Verón. A perspectiva sistêmico-discursiva possibilitou a exploração de elementos comunicacionais, articulados em um modelo teórico voltado para a compreensão da estratégia.

Palavras-chave: organização; discurso; estratégia.

Abstract: A communicational approach for organizational strategy - This paper presents a communicational approach to the organizational strategy. As a common object of administrative and economic strategies, the procedure was investigated from an epistemic-methodological perspective which emphasizes communication. Grounded in Niklas Luhmann's systems theory in the social dimension of language, as well as in Eliseo Verón's discursive approach, the systemic-discursive perspective enables the exploration of communication elements, which are articulated in a theoretical model focused on understanding the organizational strategy.

Keywords: organization; discourse; strategy.

\section{Introdução}

Tradicionalmente considerada um objeto da administração e da economia, a estratégia organizacional é o foco da reflexão apresentada neste artigo. No entanto, nossa leitura é fundamentada na comunicação e tem como objetivo articular um modelo teórico que possibilite a compreensão da estratégia a partir de elementos comunicacionais. Com isso, pretendemos um deslocamento do olhar, buscando um desentranhamento do que há de propriamente comunicacional nesse objeto de interface (BRAGA, 2004) que é a estratégia organizacional.

A estratégia organizacional já foi objeto de discussão e de investigação de estudos realizados por pesquisadores brasileiros ligados à área da comunicação, como Bueno (2009); Bulgacov e 
Marchiori (2010, 2011); Kunsch (2009) e Reis, Marchiori e Casali (2010) e, mais recentemente, por Lima $(2014,2015)$. Nossa reflexão procura discutir a relação entre comunicação e estratégia indo além da compreensão da primeira como um elemento da administração estratégica ou de sua prática. Assim como Pérez (2008, 2012) e Pérez e Massoni (2009), postulamos que a comunicação pode constituir um locus ideal para (re)pensar a estratégia, revelando dimensões pouco exploradas por abordagens econômicas e administrativas.

A perspectiva epistêmico-metodológica que ampara nossa reflexão foi originalmente proposta por Seidl (2007), com base na teoria dos sistemas de Niklas Luhmann (1997a; 1997b; 2005; 2006; 2011), na filosofia da linguagem de Ludwig Wittgenstein (1979) e na concepção social da linguagem de Jean-François Lyotard (1999; 2002). À proposta de Seidl (2007), agregamos a abordagem discursiva de Eliseo Véron (1980; 1996; 2004), constituindo o que convencionamos chamar de perspectiva sistêmico-discursiva.

Um dos principais fundamentos dessa perspectiva é a compreensão dos sistemas sociais como unidades operacionalmente fechadas e interativamente abertas, que têm a comunicação como sua operação essencial. A diferença entre o sistema social e o ambiente é (re)produzida continuamente através da comunicação, garantindo a manutenção do sistema e procurando reduzir sua complexidade. A diferenciação dos sistemas sociais parece seguir um movimento reflexivo e recursivo, em que o sentido é construído em uma rede de comunicações, que opera de forma circular, autopoiética ${ }^{1}$ e operacionalmente fechada.

No momento da realização de processos de comunicação, é construída uma diferença entre comunicação e meio (sistema e ambiente). A comunicação é responsável pelo desenvolvimento de uma lógica própria de conexão com a comunicação seguinte, formando uma rede que inventa sua própria memória e diferencia os sistemas sociais. Os sentidos, portanto, circulam na rede, e constituem o horizonte operativo dos sistemas sociais, que apesar de operacionalmente fechados, não estão isolados.

A linguagem possibilita acoplamentos estruturais ${ }^{2}$ com indivíduos e outros sistemas, permitindo que elementos externos a eles sejam incorporados, mantendo a autorreferencialidade na construção dos sentidos. Ou seja, o sistema tem contato com o mundo externo, mas constrói o sentido segundo suas referências internas. Isso garante a identidade e mantém a diferenciação sistema/ambiente.

Nesse contexto, os discursos são compreendidos como efeitos de sentidos construídos nos sistemas sociais. Podem ser entendidos também como lances de linguagem ou enunciados que, em jogos de linguagem, materializam os sentidos que circulam nos sistemas.

1 O adjetivo "autopoiética" é utilizado para designar uma estrutura (sistema) em que ocorre a autopoiese, ou seja, a capacidade de autorreproduzir-se exclusivamente através de seus próprios elementos e operações internas. No caso dos sistemas sociais, a comunicação e a rede de comunicações formada no interior do sistema constituem o elemento fundamental para a autorreprodução e são por ele responsáveis (LUHMANN, 2011).

2 O sistema, segundo Luhmann (2011), se relaciona com o meio circundante através do acoplamento estrutural. O acoplamento considera o fechamento operacional e a relação com o ambiente a partir dos mecanismos internos de operação do sistema. Assim como a diferença entre ele e o ambiente é produzida pelo próprio sistema, as relações que este estabelece com o ambiente também são resultados dessas operações internas. 
Esses lances parecem estar submetidos a regras e acordos, explícitos ou tácitos, que são intrínsecos à condição de fechamento dos sistemas sociais. Além disso, os lances configuram um contexto social e com isso assumem posições, expectativas e disputas.

A teoria do discurso de Verón (1980, 1996, 2004) é construída sobre uma hipótese de defasagem entre as noções de "produção" e de "reconhecimento", guardando semelhanças com as noções de emissão e de recepção da teoria da comunicação. A problemática da comunicação, para o autor, está justamente nessa defasagem. Há uma não linearidade entre produção e reconhecimento, o que implica num discurso que nunca produz um efeito único, mas um campo de efeitos. A não linearidade expressa uma circulação de sentidos em um sistema em desequilíbrio. Os discursos circulam entre condições de produção e condições de reconhecimento, formando uma rede de semiose (VÉRON, 1980, 1996, 2004) em que um discurso é condição produtiva de outro. Assim, a análise não pode considerar o objeto em si mesmo. Ela precisa considerar a relação do objeto com aspectos determinados das condições, buscando as pistas ou marcas dessas nos textos analisados.

A perspectiva sistêmico-discursiva contribui para direcionar a atenção para a dimensão social do discurso, não ignorando o sujeito, mas deslocando o foco para a diferença sistema/ ambiente e para a dinâmica social da construção de sentidos. A concepção da organização, sob essa mesma perspectiva, como um sistema social operacionalmente fechado e interativamente aberto, que tem a comunicação como sua operação essencial, leva aos elementos comunicacionais que são articulados no modelo teórico: a decisão, a identificação, a institucionalização, a mediação, a cultura organizacional e a comunicação organizacional.

A seguir, apresentamos o modelo teórico que possibilita a leitura comunicacional da estratégia. Ele é configurado em torno de uma noção central, que denominamos rede decisória comunicacional, articulando os elementos comunicacionais acima mencionados.

\section{A rede decisória comunicacional}

Reconhecendo que estratégias são formadas em e por decisões (ANSOFF, 1983; CHANDLER, 1998; MINTZBERG; WATERS, 1985; PÉREZ, 2008) e que decisões são uma forma de comunicação responsável pela constituição e manutenção dos sistemas organizacionais, as estratégias organizacionais podem ser compreendidas como decisões voltadas para a (re)produção da diferença entre organização e ambiente. Fundamentados pela teoria dos sistemas de Luhmann (2005, 2011), assumimos a distinção sistema organizacional/ambiente como ponto de partida para a construção de nosso modelo. Essa distinção, criada a partir de observações do próprio sistema, precisa ser continuamente (re)produzida, de forma reflexiva e recursiva pela comunicação.

A busca por diferenciação em abordagens econômicas ou administrativas pode ser discutida a partir da noção de vantagem competitiva. Na leitura comunicacional, compreendemos a diferenciação a partir do processo de identificação e da noção de identidade. 
Partindo de uma concepção relacional da identidade, consideramos que ela é construída permanentemente na relação com o ambiente. Assim, parece haver um esforço contínuo dos sistemas em estabelecerem seus limites em relação ao ambiente, na medida em que aumentam sua própria complexidade, em um processo autopoiético.

A distinção entre os sistemas organizacionais e o ambiente pode ser compreendida através das fronteiras simbólicas, estabelecidas pelos próprios sistemas na relação com o ambiente. Trata-se, portanto, de um processo comunicacional em que são construídos sentidos sobre o que faz e o que não faz parte desses sistemas.

No caso dos sistemas organizacionais, essa comunicação, que define as fronteiras ou marcações simbólicas, assume a forma de decisões. Assim, é possível dizer que as estruturas dos sistemas organizacionais são originalmente construídas e mantidas pelas decisões. Instantâneas e contingentes, as decisões como elementos centrais dos sistemas organizacionais, são constantemente (re)produzidas, formando uma rede voltada para criação e manutenção da distinção. Nessa perspectiva podemos assumir, portanto, que as organizações existem enquanto decidem.

A decisão, reforçamos, não é aqui compreendida como resultado de uma racionalidade absoluta, mas como fruto de uma racionalidade múltipla, de diferentes expressões da razão, "associadas a sentimentos e emoções, com espaço particular para a intuição" (LEITÃO, 1997, p. 91). Assim, no ambiente organizacional, a decisão parece ser influenciada por uma diversidade de fatores que incluem, além dos aspectos materiais, a cultura, as relações de poder e, também, as relações afetivas. Podemos pensar na decisão como a "ponta do iceberg", que encerra, por sua natureza, um conjunto amplo e complexo de motivações e, consequentemente, de representações das realidades organizacionais.

A informação é geralmente considerada um dos principais insumos para o processo decisório. Entretanto, a perspectiva sistêmico-discursiva faz avançar nossa compreensão, pois é a comunicação que leva a uma decisão, e não a informação pura e simples. No momento em que uma decisão é tomada, é possível pensarmos que houve um efeito de sentido.

Os indivíduos ou sistemas decidem a partir de efeitos de sentido e de processos de reconhecimento. Conforme lembram Rodrigues e Neves (2012, p. 47), com base em Luhmann, "o sentido determina o horizonte operativo dos sistemas sociais".

Assim, as decisões podem ser consideradas efeitos de sentidos, ou, em outras palavras, discursos. Elas adquirem sentido no interior dos sistemas em que são produzidas e podem ver seu sentido multiplicado no momento em que há interação entre sistemas. Dessa forma, parece possível dizer que a estratégia é configurada em um conjunto de discursos/decisões, (re)construídos permanentemente, através da produção e do reconhecimento de textos sobre as diferenças da organização, considerando-se seu ambiente. Essa (re)construção se 
dá através da interação e da perturbação entre sistemas, levando a uma multiplicação ${ }^{3}$ de sentidos que é, por sua natureza, incontrolável. Isso por um lado, dificulta a compreensão e a gestão, por outro, ressalta a dinamicidade do processo estratégico.

Uma decisão considerada individualmente apresenta uma fragilidade devido ao paradoxo que ela guarda em seu interior. Toda decisão informa sobre uma seleção, em que uma alternativa é escolhida em detrimento de outras. Essa fragilidade é atenuada pelo conteúdo de decisões posteriores. Ou seja, uma decisão geralmente é conectada a outra, como forma de reafirmação e de redução das incertezas inerentes aos processos decisórios. A ligação entre as decisões ocorre em uma rede, na qual uma decisão funciona como premissa para outras, fazendo com que a justificativa para elas esteja na própria rede.

A noção de rede que fundamenta nosso modelo pode também ser associada a concepções do processo comunicacional (FAUSTO NETO, 2008; LYOTARD, 2002; RESTREPPO, 1995) e da mesma forma, está presente na abordagem discursiva de Verón (1980,1996). Para este autor (VERÓN, 1980, p. 193), “a semiose social é uma rede significante infinita", em que os discursos se relacionam, constituindo suas próprias condições produtivas. Com base nas noções de rede de decisões e de rede de semiose, chegamos à proposta da rede decisória comunicacional.

Ela é um espaço de circulação, multiplicação e disputa de sentidos, que constituem diferentes campos de efeitos e consequentemente, diferentes condições produtivas para os discursos/decisões. Seu efeito, portanto, pode ser analisado quando ele constitui parte das condições de produção de um discurso/decisão subsequente. Importa ressaltar que a rede, por sua natureza, é um espaço não linear e transitório. As conexões entre as decisões são estabelecidas, sendo possível de alguma forma, perceber a relação entre elas. Mas, devido à sua natureza comunicacional, a rede é um espaço transitório, instável e contingente, precisando ser constantemente (re)produzido

Essa mesma natureza revela aspectos afetivos, emotivos e intuitivos, envolvidos nos processos decisórios e na constituição sistêmica da organização. Considerando que os sistemas psíquicos ou as consciências são pressupostos para a existência dos sistemas organizacionais, podemos dizer que esses aspectos também constituem a rede decisória comunicacional e estão nela representados ${ }^{4}$.

Na perspectiva sistêmico-discursiva, a rede decisória comunicacional pode ser compreendida como várias redes em relação. Sendo o sentido uma construção particular de cada sistema, assim parece possível pensar em redes internas aos sistemas organizacionais

3 A noção de multiplicação de sentidos é fundamentada em Luhmann (2011), para quem a metáfora da transferência ou transmissão não é adequada para a explicação do processo comunicacional. Conforme o autor (2011, p. 294), "a comunicação é uma sucessão de efeitos multiplicadores". Assim, quando nos referimos à multiplicação de sentidos, não consideramos a noção matemática que pressupõe a manutenção de um mesmo objeto ou a soma finita de números iguais. A multiplicação é compreendida como uma sucessão de efeitos do processo comunicacional, que gera multiplicidade de sentidos.

4 Importa lembrar que consideramos indivíduos como sistemas psíquicos, que podem estar acoplados a uma organização (sistema social). Sistemas psíquicos e sociais se interpenetram e podem apresentar uma coevolução (LUHMANN, 2011). 
e também em redes externas, constituídas nos ambientes externos, ou seja, nos sistemas sociais aos quais as organizações se acoplam e com os quais interagem.

Essas redes são (re)produzidas nas relações entre sistemas. Seidl e Becker (2006) chamam atenção para os "rótulos", que podem ser palavras ou frases que circulam entre diferentes discursos, assumindo diferentes sentidos, de acordo com os sistemas que os produzem e os reconhecem. A rede decisória comunicacional se constitui como um espaço autônomo de interação e de circulação, multiplicação e disputa de sentidos, que são apropriados e reciclados em operações internas dos sistemas. Essa apropriação parece seguir a dinâmica de jogos de linguagem, que particularizam sentidos em acordos tácitos e/ou explícitos.

No contexto da rede decisória comunicacional é que pensamos as decisões. Interconectadas, elas formam a estratégia organizacional em uma busca constante por autofortificação dos sistemas organizacionais. Produzidas no interior desses sistemas como condição de sua própria existência, as decisões sofrem influência do ambiente e são construídas em uma permanente tensão entre sistema e ambiente. Na relação com o ambiente ocorrem os processos de identificação e a construção das identidades, ao mesmo tempo em que depende das decisões, no âmbito operacionalmente fechado dos sistemas organizacionais, a construção das identidades depende do ambiente e das interações ou acoplamentos com outros sistemas.

O sistema observa, seleciona e processa informações do ambiente de forma contínua, autofortificando-se, procurando reduzir a complexidade externa e, ao mesmo tempo, aumentando sua própria complexidade. Assim, é possível dizer que a alteridade/ambiente se faz presente na identidade e que esta última está presente no ambiente. Construída em relações ou interações, a identidade depende diretamente da comunicação. Conforme Hall (2009), as identificações operam através de um trabalho discursivo, em que são marcadas as fronteiras simbólicas e são produzidos "efeitos de fronteiras", que podemos entender como efeitos de sentido.

Parece natural pensar que as organizações busquem deliberadamente demarcar e controlar suas fronteiras sistêmicas, ou seja, gerenciar suas identificações. No entanto, ao longo de suas trajetórias, algumas decisões podem contribuir para desvios e identificações, não necessariamente desejadas ou planejadas pelas organizações, que têm efeito sobre a demarcação de suas fronteiras. Assim, é possível dizer que a estratégia também apresenta uma dimensão incontrolável, que é inerente à sua natureza comunicacional. Nesse sentido é que entendemos a estratégia como um conjunto de decisões voltadas para construir, manter e controlar, na medida do possível, as identificações e as identidades das organizações.

A comunicação em forma de decisões assume diferentes objetivos, não se restringindo apenas a afirmação de uma identidade. No entanto, parece haver em todas as comunicações, fruto da permanente tensão sistema/ambiente, ingredientes ou marcas que, em maior ou menor grau, revelam o esforço de diferenciação. O que procuramos destacar no modelo proposto é justamente essa dimensão estratégica, entendendo que 
a estratégia organizacional pode ser desvendada com base nesses aspectos que criam e reforçam a distinção fundamental do sistema organizacional.

Em nossa leitura, a natureza comunicacional da estratégia é revelada em seu estado de permanente (re)construção, no âmbito da rede decisória comunicacional. É essa natureza que indica os processos de institucionalização e de mediação da estratégia, bem como sua relação com a cultura e a comunicação organizacional.

A institucionalização revela o poder de convocação de um universo autorreferenciado de sentidos, que leva as organizações e suas estratégias a (re)produzirem suas próprias estruturas e aquelas dos sistemas com os quais se relacionam. A rede decisória comunicacional parece ser responsável por legitimar e institucionalizar as decisões da estratégia organizacional, contribuindo para formação de um isomorfismo institucional em torno da própria estratégia. A tendência à homogeneização pode ocorrer devido a pressões políticas e culturais, devido à busca por reduzir as incertezas associadas às decisões ou à legitimação de práticas profissionais.

Considerar a institucionalização não significa ignorar a existência de movimentos de inovação. Os sistemas organizacionais como instâncias autônomas de mediação, ou seja, de apropriação e de recodificação de sentidos, guardam sua capacidade de inovação. A mediação pode resultar em decisões que venham a configurar inovações, reforçando as fronteiras sistêmicas e a identidade. Por sinal, a transitoriedade e a contingência das estruturas sistêmicas, em uma leitura comunicacional, parecem pressionar por mudanças e por inovações, que podem ser compreendidas como novas configurações de sentido no interior das estruturas comunicacionais dos sistemas.

A rede decisória comunicacional - na medida em que constitui as condições produtivas das decisões estratégicas, que institucionaliza nas relações de poder um "repertório" de decisões ou campos de efeitos - é também um espaço de mediação das decisões ou discursos estratégicos. Nesse contexto, entende-se a mediação como construções culturais e simbólicas e as apropriações e recodificações, como responsáveis pela criação dos sentidos e pelas decisões (efeitos de sentidos). A mediação, conforme propõe Martín-Barbero (2006), é atravessada por racionalidades ligadas à economia, à política e ao imaginário, e também pelo cotidiano e pela cultura.

A estratégia seria então mediada na rede decisória comunicacional, quando as organizações buscam construir suas decisões sob a influência de outras anteriores ou quando perturbadas por decisões de outros sistemas que estão em seu ambiente. Da mesma forma, determinados saberes, hábitos e técnicas que circulam na rede, parecem conferir a institucionalidade necessária para a construção das estratégias. Assim, entendemos que as diferentes apropriações desses saberes, hábitos e técnicas pelas organizações, podem ser observadas para aprofundar a compreensão das estratégias organizacionais.

A mediação nos ambientes organizacionais, assim como em sua concepção original (MARTIN-BARBERO, 2006), revela profunda imbricação entre a comunicação e 
a cultura. A rede decisória comunicacional, no interior das organizações, também pode ser compreendida como uma teia de significados (GEERTZ, 2003), como representação ou representações da cultura organizacional, que deixam "marcas" nos discursos da estratégia, revelando as identidades organizacionais. Ao mesmo tempo em que representa a cultura organizacional, a rede decisória comunicacional pode ser considerada responsável por sua construção. Assim, a cultura é (re)construída e também revelada na rede e pode ser considerada condição produtiva das decisões estratégicas, além de um dos elementos centrais da leitura comunicacional da estratégia.

No contexto da rede decisória comunicacional, a comunicação organizacional pensada a partir de abordagens sistêmicas, complexas e discursivas (BALDISSERA, 2008; CURVELLO 2009; DEETZ, 2001; FAIRHURST; PUTMAN, 2010; FAUSTO NETO, 2008; MARSHACK; GRANT, 2008; RESTREPPO, 1995), emerge como um elemento processual de constituição, de negociação, de representação dos sistemas organizacionais e de suas estratégias organizacionais, através da circulação, da multiplicação e da disputa de sentidos. Esses sentidos são materializados em discursos formais e informais, controlados ou não, que atravessam as mais diversas práticas comunicacionais, envolvendo desde conversas e reuniões informais, até iniciativas planejadas de comunicação.

De modo sintético, é possível dizer que a estratégia organizacional é constituída, negociada e representada, através da processualidade da comunicação organizacional, em um conjunto de decisões voltadas para a construção e manutenção das fronteiras sistêmicas. Essas decisões são produzidas, reconhecidas e interconectadas na rede decisória comunicacional. Ela, por sua vez:

1. (re)produz processos de identificação voltados para a demarcação das fronteiras sistêmicas;

2. legitima e institucionaliza em relações de poder, um "repertório" de decisões ou efeitos de sentido;

3. media os sentidos através de apropriações e recodificações;

4. representa e ao mesmo tempo, constrói a cultura organizacional.

A figura 1 a seguir, representa essa síntese. No centro dela está a rede decisória comunicacional, constituída na interconexão das decisões e através da processualidade da comunicação organizacional. Os processos de identificação, de institucionalização e de mediação, representados no núcleo central, ocorrem na rede e contribuem para a sua constituição. A cultura organizacional, como condição produtiva das decisões, deixa marcas sobre a rede (setas brancas) e também é construída por ela (setas verdes). Por fim, a estratégia organizacional, resultante das processualidades sistêmicas, é responsável pela demarcação das fronteiras entre a organização e o ambiente. O sistema organizacional fica assim representado pelo esforço estratégico de distinção em relação ao ambiente externo, que é constituído por outros sistemas e redes decisórias. 


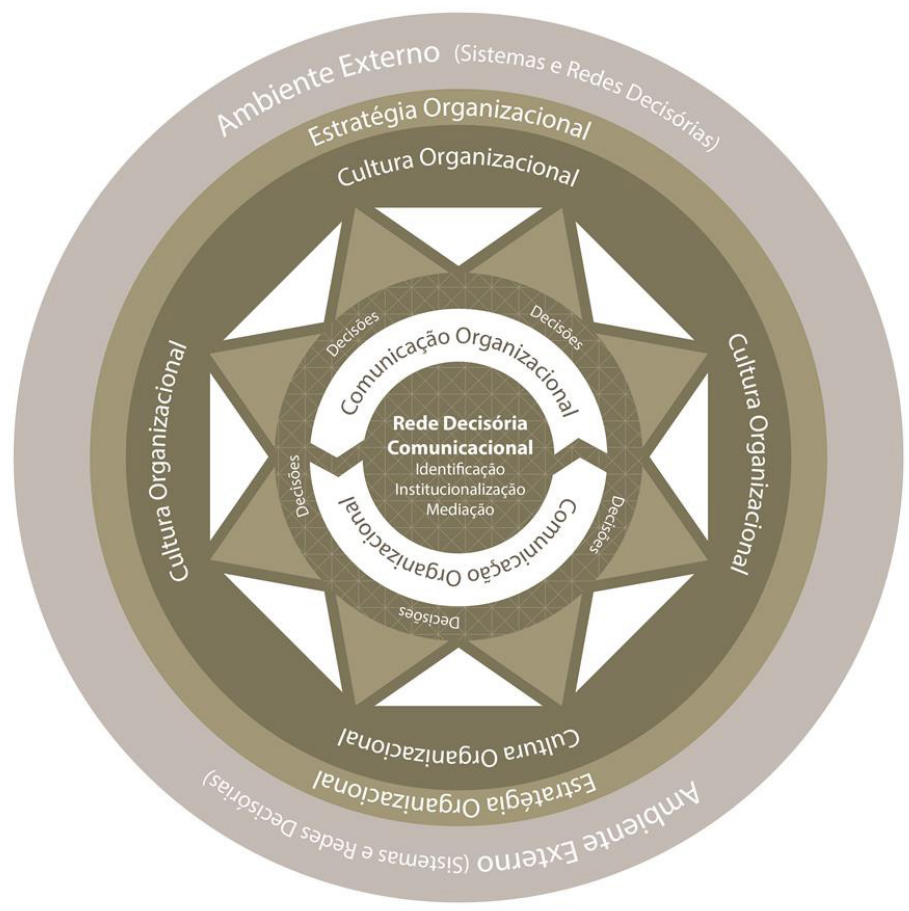

Fig.1. Rede decisória comunicacional

Fonte: Elaborada pelo autor.

\section{Considerações}

Com este trabalho, exploramos um caminho para pensar a estratégia organizacional e não necessariamente para fazer a estratégia. Assim, o modelo proposto pretende ser um estímulo para refletir, discutir e revelar os sentidos da estratégia organizacional a partir da processualidade e da materialidade da comunicação organizacional.

A diversidade de textos produzidos nos sistemas organizacionais, em processos controlados ou não, constrói e representa decisões responsáveis pela gênese e pela manutenção desses sistemas. Pensar a estratégia com base no modelo aqui apresentado, consiste em abordar discursivamente os textos em busca das diferentes representações das decisões, que conforme vimos, estão no cerne das estratégias organizacionais. Não se trata de uma reflexão sobre estratégias de comunicação, mas de um exercício cujo objetivo central é desvendar os elementos comunicacionais da estratégia.

Importa ratificar que a estratégia a que nos referimos é aquela construída com base em um leque abrangente de decisões, que podem ter impacto sobre a sobrevivência das organizações e que não se restringem, apesar de incluírem, as decisões relativas às práticas 
de comunicação. Essas decisões envolvem um amplo escopo de movimentos, desde a mobilização de recursos, a definição de objetivos, metas e percursos, até as respostas aos constantes desafios impostos pelo ambiente.

A comunicação organizacional, da forma como pressupomos, em suas diversas manifestações, (re)produz sentidos na permanente tensão organização/ambiente. Essa concepção é fundamentada na noção de organização como um sistema social que se distingue e se (re)produz a partir da comunicação em forma de decisão (LUHMANN, 2005).

Outro aspecto central dessa noção é a relação com o ambiente, que deixa de ser (uni)causal, conforme Crubellate e Machado (2010), em que o ambiente determina a organização e passa a considerar a relação de codependência entre organização e ambiente. Dessa forma, a comunicação organizacional não é compreendida em modalidades exclusivamente internas ou externas, mas sim, como uma rede de sentidos construída em processos que apresentam dimensões internas e externas, ou seja, as práticas e produtos dos processos comunicacionais são atravessados por elementos internos e externos às fronteiras organizacionais, da mesma forma que podem produzir efeitos interna e externamente.

O modelo da rede decisória comunicacional, portanto, pressupõe a multidimensionalidade da comunicação organizacional e o aspecto ontológico de sua relação com o ambiente. Sua principal aposta é que a leitura de textos produzidos no âmbito da comunicação organizacional revele as decisões e, consequentemente, as estratégias das organizações, indicando que há elementos comunicacionais nos processos de formação das estratégias das organizações: a identificação, a institucionalização, a mediação, a cultura organizacional, além dos sentidos que circulam nas decisões, são construídos nos processos comunicacionais.

Considerando que a decisão em forma de comunicação está na gênese das organizações, o modelo assume ainda, de forma coerente com seus pressupostos teóricos, que a leitura comunicacional pode ser realizada em organizações já estabelecidas, independentemente de seu porte ou setor de atuação, assim como para, a partir de suas decisões originais, compreender as estratégias de novas organizações.

Victor Márcio Laus Reis Gomes é professor e pesquisador do Programa de Pós-Graduação em Comunicação da Universidade Católica de Brasília.

victorlaus@gmail.com

\section{Referências}

ANSOFF, I. H. Administração estratégica. São Paulo: Atlas, 1983. 
BALDISSERA, R. Por uma compreensão da comunicação organizacional. In: SCROFERNEKER, C. M. A. (Org.). O diálogo possível: comunicação organizacional e paradigma da complexidade. Porto Alegre: EDIPUCRS, 2008. p. 31-50.

BRAGA, J. L. Os estudos de interface como espaço de construção do Campo da Comunicação. Contracampo, n. 10/11, v. 2, p. 219-235, 2004.

BUENO, W. C. Comunicação empresarial: políticas e estratégias. São Paulo: Saraiva, 2009.

BULGACOV, S.; MARCHIORI, M. Estratégia como prática: a construção de uma realidade social em processos de interação organizacional. In: MARCHIORI, M. (Org.). Comunicação e organização: reflexões, processos e práticas. São Caetano do Sul: Difusão, 2010. p. 149-166.

. Strategy as communicational practice in organizations. In: PRE-CONFERENCE IN STRATEGIC COMMUNICATION - ICA CONFERENCE, 2011, Boston. Strategic Communication Issues. Washington, DC: Internationational Communication Association, 2011. p. 1-22.

CHANDLER, A. D. Strategy and structure: chapters in the history of the industrial enterprise. Cambridge: MIT Press, 1998.

CURVELLO, J. J. A. A perspectiva sistêmico-comunicacional das organizações e sua importância para os estudos da comunicação organizacional. In: KUNSCH, M. M. K. (Org.). Comunicação organizacional: histórico, fundamentos e processos. São Paulo: Saraiva, 2009. v.1, p. 91-106.

CRUBELLATE, J. M.; MACHADO, H. P. V. Organizações e ambiente organizacional: uma abordagem neofuncionalista. In: MARCHIORI, M. (Org.). Comunicação e organização: reflexões, processos e práticas. São Caetano do Sul: Difusão, 2010. p. 51-68.

DEETZ, S. Conceptual foundations. In: JABLIN, F. M.; PUTNAM, L. L. (Org.). The new handbook of organizational communication: advances in theory, research and methods. Thousand Oaks: Sage, 2001. p. 3-46.

FAIRHURST, G. T.; PUTNAM, L. As organizações como construções discursivas. In: MARCHIORI, M. (Org.). Comunicação e organização: reflexões, processos e práticas. São Caetano do Sul: Difusão, 2010. p. 103-148.

FAUSTO NETO, A. Comunicação das organizações: da vigilância aos pontos de fuga. In: OLIVEIRA, I. L.; SOARES, A. T. N. Interfaces e tendências da comunicação no contexto das organizações. São Caetano do Sul: Difusão, 2008.

GEERTZ, C. A interpretação das culturas. São Paulo: LTC, 2003.

HALL, S. Quem precisa da identidade? In: SILVA, T. T. (Org.). Identidade e diferença: a perspectiva dos Estudos Culturais. Petrópolis: Vozes, 2009.

HENDRY, J. Strategic decision making, discourse and strategy as social practice. Journal of Management Studies, v. 37, n. 7, p. 955-977, 2000.

KUNSCH, M. M. K. Planejamento estratégico da comunicação. In: KUNSCH, M. M. K. (Org.). Gestão estratégica da comunicação organizacional e relações públicas. São Caetano do Sul: Difusão, 2009.

LEITÃO, S. P. Para uma nova teoria da decisão organizacional. RAP, Rio de Janeiro, v. 31, n. 2, p. 91-107, mar./abr. 1997.

LIMA, F. P. A dimensão comunicacional da estratégia: a estratégia organizacional como prática comunicativa na Secretaria de Estado de Fazenda de Minas Gerais. Tese (Doutorado em Ciências 
da Comunicação) - Escola de Comunicações e Artes, Universidade de São Paulo, São Paulo, 2014.

A estratégia como prática de comunicação. In: MARQUES, A.; OLIVEIRA, I. L. (Orgs). Comunicação Organizacional: dimensões epistemológicas e discursivas. 1ed.Belo Horizonte: FAFICH/ UFMG, 2015, v. , p. 135-143.

LUHMANN, N. Por que uma "teoria dos sistemas"? In: NEVES, C. E. B.; SAMIOS, E. M. B. (orgs.). Niklas Luhmann: a nova Teoria dos Sistemas. Porto Alegre: Ed. Universidade/UFRGS, Goethe-Institut/ ICBA, 1997a.

. Novos desenvolvimentos na teoria dos sistemas. In: NEVES, C. E. B.; SAMIOS, E. M. B. (Org.). Niklas Luhmann: a nova Teoria dos Sistemas. Porto Alegre: Ed. Universidade/UFRGS; Goethe-Institut/ ICBA, $1997 \mathrm{~b}$.

. The paradox of decision making. In: SEIDL, D. BECKER; K. H. (Org.). Niklas Luhmann and Organization Studies. Copenhagen: Copenhagen Business School Press, 2005.

A improbabilidade da comunicação. Lisboa: Vega, 2006.

Introdução à teoria dos sistemas. Petrópolis: Vozes, 2011.

LYOTARD, J. F. La diferencia. Barcelona: Gedisa, 1999.

. A condição pós-moderna. Rio de Janeiro: José Olympio, 2002.

MARSHAK, R. J.; GRANT, D. Organizational discourse and new organization development practices. British Journal of Management, v. 19, p. 7-19, 2008.

MARTIN-BARBERO, J. Dos meios às mediações: comunicação, cultura e hegemonia. Rio de Janeiro: Ed. UFRJ, 2006.

MINZBERG, H.; WATERS, J. A. Of strategies, deliberate and emergent. Strategic Management Journal, 6, p. 257-272, 1985.

PALLI, P.; VAARA, E.; SORSA, V. Strategy as text and discursive practice: a genre-based approach to strategizing in city administration. Discourse \& Communication, v. 3, n. 3, p. 303-318, 2009.

PÉREZ, R. A. Estrategias de comunicación. Barcelona: Ariel, 2008.

. Pensar la estrategia. Buenos Aires: La Crujía, 2012.

PÉREZ, R. A.; MASSONI, S. Hacia una teoría general de la estrategia. Barcelona: Ariel, 2009.

REIS, M. C.; MARCHIORI, Marlene; CASALI, A. M. A relação comunicação-estratégia no contexto das práticas organizacionais. In: MARCHIORI, M. (Org.). Comunicação e organização: reflexões, processos e práticas. São Caetano do Sul: Difusão, 2010. p. 167-187.

RESTREPPO, M. J. Comunicación para la dinámica organizacional. Signo y Pensamiento, n. 26, p. 91-96, 1995.

RODRIGUES, L. P.; NEVES, F. M. Niklas Luhmann: a sociedade como sistema. Porto Alegre: EDIPUCRS, 2012.

SEIDL, D. General strategy concepts and the ecology of strategy discourses: a systemic-discursive perspective. Organization Studies, v. 28, n. 2, p. 197-218, 2007.

SEIDL, D.; BECKER, K. H.. Organizations as distinction generating and processing systems: Niklas's 
Luhmann contribution to organization studies. Organization, v. 13, n. 9, p. 9-35, 2006.

VERÓN, E. A produção de sentido. São Paulo: Cultrix; Ed. da USP, 1980.

La semiosis social: fragmentos de uma teoria de la discursividad. Barcelona: Gedisa, 1996.

Fragmentos de um tecido. São Leopoldo: Ed. Unisinos, 2004.

WITTGENSTEIN, L. Investigações Filosóficas. São Paulo: Abril Cultural, 1979.

Artigo recebido em novembro de 2015

e aprovado em março de 2016. 